\title{
Estimation of the Radio Channel Parameters from a Circular Array with Directional Antennas
}

\author{
Annika Böttcher, Peter Vary \\ Inst. of Communication Systems and Data Processing \\ RWTH Aachen University, Germany \\ \{boettcher|vary\}@ind.rwth-aachen.de
}

\author{
Christian Schneider, Milan Narandzic, Reiner S. Thomä \\ Institute of Information Technology \\ Ilmenau University of Technology, Germany \\ christian.schneider@tu-ilmenau.de
}

\begin{abstract}
Channel sounding, channel modeling and their parameter extraction in particular considering MIMO configurations, plays an important role for todays realistic wireless system design and evaluation. The analysis of the gathered channel sounding data has to match the specifics in the measurement system configuration. Within this contribution directional antenna arrays are used at both sides of the measured link. Furthermore, the measurement system applies automatic gain control between subsequent MIMO snapshots and additionally within the MIMO matrix. Both features lead to improved measurement accuracy. A new extension of existing power and delay domain analysis procedures considering the automatic gain control within the MIMO matrix is introduced and evaluated. The results show that delay spread and K-factor are more affected, while shadow fading and cross polarization ratios are almost not.
\end{abstract}

\section{INTRODUCTION}

For the evaluation of multi antenna system designs, mobile radio measurements of the target environment are needed. On the one hand measurement data can directly be used for various evaluations, but on the other hand channel models which reflect the natural stochastic behavior of the radio environment are of particular importance. Besides the proper definition of the measurement configuration, a second critical point arises from the analysis and estimation of the channel model parameters. Therefore, channel measurements have to be processed in predefined ways which are in line with the considered channel model concept. But thereby the specific measurement setup and more important the antenna configuration have to be taken into account.

In this paper different approaches for the estimation of the large scale parameters (LSP) for the WINNER II channel model [1] are presented and analyzed. In general most of the known channel sounder systems allow automatic gain control (AGC) switching between consecutively measured channel impulse responses (CIR). Usually, in the case of MIMO measurements the AGC is constant for one snapshot and all MIMO CIRs. The RUSK TUI FAU channel sounder [2], which we have used for our measurements, provides the possibility to switch the AGC between the MIMO channels even within one snapshot. This kind of AGC switching is very profitable because this feature holds all the dynamic of the channel and thus affords a more precise measurement. Due to the

This work has been supported by the UMIC Research Center, RWTH Aachen University. fading caused by the multipath propagation and especially the characteristics of our antenna at receiver side which is a uniform circular array (UCA) with directional antenna patches, the MIMO channel can vary extremely depending on the selection of the antenna patch compared to a UCA with omnidirectional antenna elements. Worth mentioning is, that the influence of the directivity of our antenna is dominant compared to the channel variation due to the fading. There exist a strong correlation between the direction of the antenna element and the AGC value, i.e. adjoined patches have nearly the same AGC value. The first estimation approach is to utilize these different AGC values of the channels to assort the measured data with the same AGC value (quantized in steps of $3 \mathrm{~dB}$ ) and to calculate the parameters for each group separately. This is a more precise estimation method than the calculation of the parameters over all channels at once and results in a better description of the parameter characteristics. The second aspect which is examined in this paper is the influence of a $20 \mathrm{~dB}$ quality threshold (QT) in regard to the parameter values and their distribution. This is an extension of the work which has been presented in [3]. The underlying measurement data sets are taken from an extensive MIMO channel sounding campaign in Ilmenau, Germany. Parts of the measurement data are free accessible via the web site [4] and can be considered as reference scenario for channel modeling and system evaluation [5].

\section{Mimo Channel Measurement CAMPAign}

The measurement and antenna setups that match the requirements of the 3GPP Long Term Evolution (LTE and LTE-A [6]) are described in detail in [5]. The channel sounding was performed at $2.53 \mathrm{GHz}$ in a band of $2 \times 45 \mathrm{MHz}$. To allow high resolution path parameter estimations, dedicated antenna arrays are used at the transmitter ( $\mathrm{Tx})$ and the receiver ( $\mathrm{Rx}$ ). On the Tx side (base station), a uniform linear array is used with 8 dualpolarized $(\mathrm{H} / \mathrm{V})$ elements, each of which consists of a stack of 4 patches in order to form a narrow transmit beam in elevation. At the mobile (passenger car), a circular array with 2 rings of 12 patches with $\mathrm{H} / \mathrm{V}$ polarizations is used. Additionally, a MIMO cube is placed on top. The mobile acts as Rx. For each of the tracks and for each measured snapshot, geo-data information based on GPS, odometer and separated distance measurements via laser is available. A typical length 
of a track is $50 \mathrm{~m}-70 \mathrm{~m}$. In total the measurement campaign covers 3 base station positions with a height of $25 \mathrm{~m}$ and $15 \mathrm{~m}$ and an additional relay point $(3.5 \mathrm{~m})$ in the middle of the scenario. In the following only the BS height of $25 \mathrm{~m}$ is considered. The intersite distance between the base stations is found to be for BS1-BS2 $=680 \mathrm{~m}, \mathrm{BS} 2-\mathrm{BS} 3=580 \mathrm{~m}$ and $\mathrm{BS} 3-\mathrm{BS} 1=640 \mathrm{~m}$. More than 20 individual tracks with more than 120 measurement runs have been performed. For more information about the measurement campaign we refer to [5] and [4].

\section{DATA AnAlysis Procedure}

Geometry based stochastic channel models like WINNER II [1] are based on large scale parameters (LSP) like the delay spread (DS), the transmission loss (TL), shadow fading (SF), the narrowband K-factor, spreads of azimuth/elevation of arrival and departure as well as the cross polarization ratio (XPR). These LSPs and their corresponding distribution functions play a fundamental role, because as global scenario dependent parameters they control the behavior of the modeled channel. In the following, the analysis steps of the LSPs and their different estimation approaches are introduced. Furthermore, a second level of analysis is introduced by the cross correlations among the aforementioned parameters.

The RUSK channel sounder provides 4-dimensional channel transfer matrices $H(t, f, s, u)$. The variable $t$ denotes the time instant of a snapshot measurement, $f$ is the frequency and $s$ is the $s$-th $\mathrm{Rx}$ and $u$ the $u$-th $\mathrm{Tx}$ antenna, respectively. For our evaluation we only use $40 \mathrm{MHz}$ in the lower band. The snapshots where a line of sight (LOS) exists and where no line of sight (NLOS) is given are separated into two groups which will be analyzed individually. The channel transfer matrices are transformed into channel impulse response matrices $h(t, \tau, s, u)$, where $\tau$ denotes delay. During the AGC dependent data acquisition the AGC values can change along the dimensions $t, s$ and $u$ of matrix $h$. In the following two different data analysis procedures are introduced. The first approach does not consider any grouping of the CIRs from one MIMO snapshot, which is denoted by $\left(\mathrm{GR}_{\mathrm{no}}\right)$. In the second one the CIRs are sorted into different AGC dependent groups, denoted by $\left(\mathrm{GR}_{\mathrm{agc}}\right)$. That means the matrices considered per MIMO subchannel with the same AGC value are grouped together and for each group the following calculations are made separately. For simplicity, the further descriptions get no specific indexing and they are valid for both ways.

Due to noise that is included in the measured channel data, a noise power estimation and subsequently a thresholding in the delay domain is applied to reduce the effect of the noise. Here, also two different approaches are observed. The first selects the maximum estimated noise level from all available CIRs and considers it as cutting level (CL). This can be applied to each group of CIRs sorted with respect to the different AGC values and to all CIRs at once where no grouping is considered. This is denoted by $\left(\mathrm{GR}_{\mathrm{agc}} \mathrm{CL}_{\max }\right)$ and $\left(\mathrm{GR}_{\mathrm{no}} \mathrm{CL}_{\max }\right)$, respectively. The second approach is the estimation of a noise threshold for each individual CIR within a MIMO channel snapshot, which is the same in the grouping and the no grouping case. This is denoted by $\left(\mathrm{GR}_{\mathrm{no}} \mathrm{CL}_{\text {ind }}\right)$. Subsequently, a $20 \mathrm{~dB}$ quality threshold (QT) is introduced additionally, i.e., only the $20 \mathrm{~dB}$ below the maximum peak of the power delay profile is considered. The calculations of the WINNER channel model parameters within this contribution always follow the same procedure, irrespective of the QT or the AGC grouping is used or not.

Now the power delay profile (PDP) $P(t, \tau)$ is needed. Therefore, the instantaneous power is estimated, a delay shift for aligning the maximum peak is introduced and the dimension of the data by averaging over the (Tx, Rx)-antenna pairs is reduced. This results in the PDP per discrete snapshot $t_{0}$ denoted by

$$
P_{t_{0}}(\tau):=P\left(t_{0}, \tau\right) .
$$

For estimating the LSPs, the PDP $P(t, \tau)$ has to be averaged over $N_{t}(l)$ snapshots, where $N_{t}$ is variable depending on the size of the stationarity interval. This results in

$$
P_{t_{l}}(\tau):=P\left(t_{l}, \tau\right)=\frac{1}{N_{t}(l)} \sum_{i=1}^{N_{t}(l)} P\left(t_{l_{i}}, \tau\right) .
$$

Here $l$ denotes the number of the stationarity interval which is given by $\left|t_{l_{N_{t}}}-t_{l_{1}}\right| \leq 10 \lambda$, where $\lambda$ is the wavelength corresponding to the carrier frequency of the system. The value of time $t$ in the middle of the interval is denoted by $t_{l}$. Based on this preprocessed data the LSP determination is executed. The calculation steps are the same whether the AGC values are taken into account or not. But considering the AGC grouping the parameters are estimated for each group and are then averaged except for the $\mathrm{K}$-factor, where the maximum is taken. In this paper only the most important steps of the parameter calculations are presented. A detailed description can be found in [7].

\section{A. RMS Delay Spread (DS)}

The first parameter which is of great interest is the root mean square (RMS) DS. It is determined based on the PDP per stationarity interval and is calculated as follows:

$$
\sigma_{t_{l}}=\sqrt{\left(\sum_{n=1}^{N_{\tau}} \tau_{n}^{2} p_{t_{l}}\left(\tau_{n}\right)\right)-\left(\sum_{n=1}^{N_{\tau}} \tau_{n} p_{t_{l}}\left(\tau_{n}\right)\right)^{2}},
$$

where $p_{t_{l}}(\tau)$ is the probability density function of $P_{t_{l}}(\tau)$.

\section{B. Transmission Loss (TL) and Shadow Fading (SF)}

Other important parameters are the TL and the corresponding SF. Based on the total power $P_{d}$ dependent on the distance $d$ the TL is calculated as

$$
\mathrm{TL}(d)=46 \mathrm{dBm}-10 \mathrm{~dB}-10 \log _{10}(P(d) / 0.001)
$$

The SF is then given by

$$
\mathrm{SF}(d)=\overline{\mathrm{TL}}(d)-\mathrm{TL}(d),
$$

where $\overline{\mathrm{TL}}(d)=B+A \cdot \log _{10}(d)$ with appropriate linear regression factors $A$ and $B$. These factors are the same for 
all AGC groups when the grouping method is considered. The difference is that for the AGC grouping method the values of the TL averaged over the different AGC groups are used. The rest of the processing holds the same as for the DS. If the $\mathrm{SF}$ were estimated considering the AGC groups, the values of the different groups have to be averaged to get one value per stationarity interval.

\section{Narrowband K-Factor}

For the estimation of the narrowband $\mathrm{K}$-factor the narrowband impulse response is needed. It is calculated as the complex sum over the delay domain of the preprocessed and delay shifted channel impulse response $\tilde{h}(t, \tau, s, u)$ with $\tau \widehat{=} \tau^{\prime}$ and is given by

$$
h_{n a r r}(t, s, u)=\sum_{i=1}^{N_{\tau}} \tilde{h}\left(t, \tau_{i}, s, u\right) .
$$

A detailed description for the estimation of the K-factor for the AGC grouping method is presented in [7]. The calculation without AGC grouping is similar, but neglects the restriction for different AGC values and uses all MIMO channels at once. Using the moment method proposed in [8] the K-factor per stationarity interval and per AGC group results in

$$
K\left(t_{l}, \mathrm{agc}\right)=\frac{1}{\frac{\mathrm{E}\left(\hat{P}_{n a r r}^{(L)}\left(t_{l}, a g c\right)\right)}{m\left(t_{l}, \mathrm{agc}\right)}-1}
$$

with

$$
m\left(t_{l}, \operatorname{agc}\right)=\sqrt{\mathrm{E}\left(\hat{P}_{\text {narr }}^{(L)}\left(t_{l}, a g c\right)\right)^{2}-\operatorname{Var}\left(\hat{P}_{\text {narr }}^{(L)}\left(t_{l}, a g c\right)\right)}
$$

and $\hat{P}_{\text {narr }}^{(L)}\left(t_{l}, a g c\right)$ is the power of the normalized values of $h_{n a r r}(t, s, u)$ per stationarity interval and AGC group. To get only one value of the $\mathrm{K}$-factor per stationarity interval the maximum value is chosen

$$
K\left(t_{l}\right)=\max _{\mathrm{agc}}\left(K\left(t_{l}, \operatorname{agc}\right)\right) .
$$

\section{Cross Polarization Ratio (XPR)}

It is differentiated between the vertical and horizontal polarization ratio which is denoted by $\mathrm{XPR}_{\mathrm{v}}$ and $\mathrm{XPR}_{\mathrm{h}}$, respectively. The XPRs are based on the total power $P_{t_{l}}$ per stationarity interval. $\mathrm{XPR}_{\mathrm{v}}$ is the ratio of the total power of all vertical-to-vertical channels and vertical-to-horizontal channels, whereas $\mathrm{XPR}_{\mathrm{h}}$ is the ratio of the total power of all horizontal-to-horizontal channels and horizontal-to-vertical channels. The calculation is described as

$$
\mathrm{XPR}_{\mathrm{v}}\left(t_{l}\right)=\frac{P_{t_{l_{\mathrm{vv}}}}}{P_{t_{l \mathrm{vh}}}}, \operatorname{XPR}_{\mathrm{h}}\left(t_{l}\right)=\frac{P_{t_{l_{\mathrm{hh}}}}}{P_{t_{l \mathrm{hv}}}}
$$

which is in line with [1].

\section{EMPIRICAL RESULTS}

In this section results from the data analysis of the LSPs are presented, using the different approaches. Mostly, an exemplary base station is chosen for the outcome description. The behavior for the other two base stations is almost equivalent. If not stated otherwise, all tracks corresponding to the used BS or BSs are considered for the evaluations.

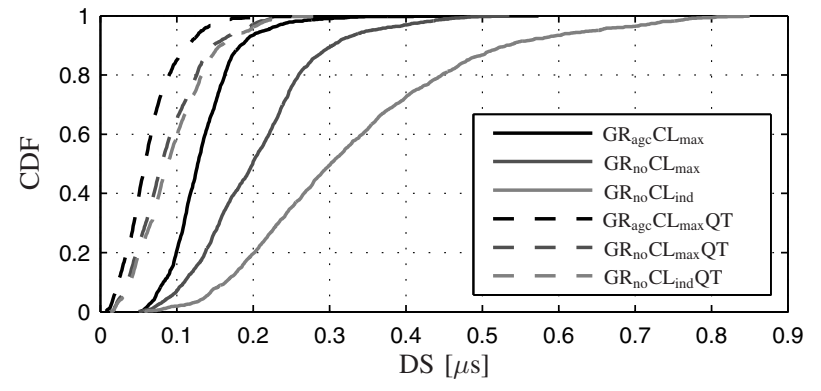

Fig. 1. CDF of the delay spread (NLOS) of BS1 for different estimation approaches.

\section{A. RMS Delay Spread (DS)}

Figure 1 shows the cumulative distribution function (CDF) of the different estimation approaches. For the calculation base station 1 and the NLOS case is chosen. What we expect is that the values for the mean and the standard deviation (std) of the DS are much smaller when the QT is introduced, because of the additional reduction of the dynamic in the PDP [9]. Furthermore the mean and standard deviation values for the DS estimated by the AGC grouping approach are expected to be smaller than without grouping. This results from the directional dependent analysis which is caused by the AGC grouping. Thus, for the calculation of the DS of each group only those multipaths and clusters are considered which belong to the particular antenna patches. This results in more similar values for the DS compared to the estimation over all antenna patches at once. In this case all multipaths and clusters which arrive at any patch are considered for the calculation. This is confirmed by the presented results in Fig. 1 and Table I. Due to the AGC dependent analysis the dynamic within measured MIMO matrices can be preserved and yields in a higher quality of the DS estimation. Hence, if the measurement method is based on the AGC switching between the MIMO channels it is indispensable to also use an AGC dependent method for the calculation of the DS.

\section{B. Transmission Loss (TL) and Shadow Fading (SF)}

For the estimation of the TL and the deductive SF all base stations are used. Table II summarizes the values $A$ and $B$ of the TL model: $\overline{\mathrm{TL}}(d)=B+A \cdot \log _{10}(d)$ for the NLOS and the LOS case. Additionally, the CDF of the SF of the NLOS case is presented in Fig. 2.

Table II and Fig. 2 show that the results for the TL and the SF are not as sensitive to the QT as the DS. This is also confirmed by Table III where the mean and the standard

\begin{tabular}{|l|c|c|c|c|}
\hline \multirow{2}{*}{} & \multicolumn{2}{|c|}{ NLOS } & \multicolumn{2}{c|}{ LOS } \\
\cline { 2 - 5 } & mean & std & mean & std \\
\hline \hline $\mathrm{GR}_{\mathrm{agc}} \mathrm{CL}_{\max }$ & $0.13 \mu \mathrm{s}$ & $0.05 \mu \mathrm{s}$ & $0.10 \mu \mathrm{s}$ & $0.02 \mu \mathrm{s}$ \\
\hline $\mathrm{GR}_{\mathrm{no}} \mathrm{CL}_{\max }$ & $0.21 \mu \mathrm{s}$ & $0.08 \mu \mathrm{s}$ & $0.11 \mu \mathrm{s}$ & $0.05 \mu \mathrm{s}$ \\
\hline $\mathrm{GR}_{\mathrm{no}} \mathrm{CL}_{\text {ind }}$ & $0.33 \mu \mathrm{s}$ & $0.15 \mu \mathrm{s}$ & $0.12 \mu \mathrm{s}$ & $0.05 \mu \mathrm{s}$ \\
\hline $\mathrm{GR}_{\mathrm{agc}} \mathrm{CL}_{\max } \mathrm{QT}$ & $0.07 \mu \mathrm{s}$ & $0.04 \mu \mathrm{s}$ & $0.05 \mu \mathrm{s}$ & $0.01 \mu \mathrm{s}$ \\
\hline $\mathrm{GR}_{\mathrm{no}} \mathrm{CL}_{\max } \mathrm{QT}$ & $0.09 \mu \mathrm{s}$ & $0.05 \mu \mathrm{s}$ & $0.05 \mu \mathrm{s}$ & $0.02 \mu \mathrm{s}$ \\
\hline $\mathrm{GR}_{\mathrm{no}} \mathrm{CL}_{\text {ind }} \mathrm{QT}$ & $0.10 \mu \mathrm{s}$ & $0.05 \mu \mathrm{s}$ & $0.05 \mu \mathrm{s}$ & $0.02 \mu \mathrm{s}$ \\
\hline WINNER II & $0.17 \mu \mathrm{s}$ & n.a. & $0.09 \mu \mathrm{s}$ & $0.08 \mu \mathrm{s}$ \\
\hline
\end{tabular}

TABLE I

VALUES FOR THE MEAN AND STANDARD DEVIATION OF THE DELAY SPREAD OF BS1 FOR DIFFERENT ESTIMATION APPROACHES. 


\begin{tabular}{|l|c|c|c|c|}
\hline & \multicolumn{2}{|c|}{ NLOS } & \multicolumn{2}{c|}{ LOS } \\
\cline { 2 - 5 } & $A$ & $B$ & $A$ & $B$ \\
\hline \hline $\mathrm{GR}_{\text {agc }} \mathrm{CL}_{\max }$ & 40.43 & 30.77 & 26.60 & 59.49 \\
\hline $\mathrm{GR}_{\text {no }} \mathrm{CL}_{\max }$ & 37.15 & 39.26 & 29.00 & 54.41 \\
\hline $\mathrm{GR}_{\text {no }} \mathrm{CL}_{\text {ind }}$ & 36.28 & 41.24 & 28.73 & 54.97 \\
\hline $\mathrm{GR}_{\text {agc }} \mathrm{CL}_{\max } \mathrm{QT}$ & 41.51 & 28.57 & 26.84 & 59.22 \\
\hline $\mathrm{GR}_{\text {no }} \mathrm{CL}_{\max } \mathrm{QT}$ & 37.37 & 38.88 & 29.10 & 54.32 \\
\hline $\mathrm{GR}_{\text {no }} \mathrm{CL}_{\text {ind }} \mathrm{QT}$ & 36.90 & 39.94 & 28.91 & 54.71 \\
\hline WINNER II & 35.70 & 36.70 & 26.00 & 50.68 \\
\hline
\end{tabular}

TABLE II

VALUES FOR THE LINEAR REGRESSION OF THE TRANSMISSION LOSS OF ALL BSS FOR DIFFERENT ESTIMATION APPROACHES.

deviation of the SF are summarized. The non-zero values of the mean SF in the AGC grouping case due to the factors $A$ and $B$, which were calculated when no AGC values are considered but hold for each single group. Nevertheless the results reflect what we expected, because using the QT results in the deletion of only very small values of the PDP, which have very low influence in terms of the total receive power calculation. The variations in the values for the NLOS case due to the AGC or no AGC grouping can be explained by the separation of different total power levels. While the total power of the non grouping method takes almost the same values (small and large ones), the variation in the values considering the AGC grouping method is much higher due to the separate noise cutting within each AGC group. Therefore, the values for the SF are additionally more spreaded around the mean value although they are averaged over the AGC groups. In the LOS case the mean value for the AGC grouping method decreases considerably compared to the non grouping method. This also can be explained by the separate examination of the total power per AGC group and by the fact that if the power of the direct path component increases, the SF decreases.

\section{Narrowband K-Factor}

The results for the K-factor analysis show the same behavior like the one of the SF. They are insensitive regarding the QT, but vary with respect to the AGC grouping. The difference is more distinct. Figure 3 presents the CDFs for the different methods for base station 1 in the LOS case. Additionally, Table IV summarizes the results.

The mean values for the AGC grouping method are much lower. This is also caused by the directional separated evaluation. Only sub-arrays of the receiver array are considered for the calculation of the K-factor per AGC group and hence, the influence of further multipath or cluster components which belong to other antenna parts (AGC groups) is reduced. Only those channels out of the MIMO matrix are taken into account

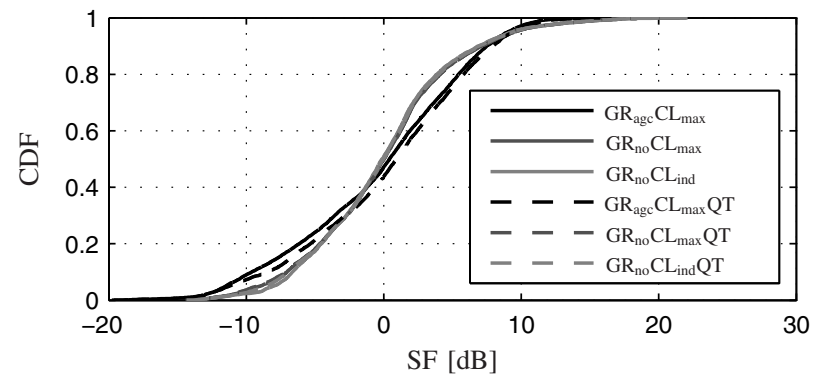

Fig. 2. CDF of the shadow fading (NLOS) of all BSs for different estimation approaches.

\begin{tabular}{|l|c|c|c|c|}
\hline \multirow{2}{*}{} & \multicolumn{2}{|c|}{ NLOS } & \multicolumn{2}{c|}{ LOS } \\
\cline { 2 - 5 } & mean & std & mean & std \\
\hline \hline $\mathrm{GR}_{\mathrm{agc}} \mathrm{CL}_{\max }$ & $-0.28 \mathrm{~dB}$ & $6.31 \mathrm{~dB}$ & $-2.40 \mathrm{~dB}$ & $7.00 \mathrm{~dB}$ \\
\hline $\mathrm{GR}_{\text {no }} \mathrm{CL}_{\max }$ & $0.00 \mathrm{~dB}$ & $5.55 \mathrm{~dB}$ & $0.00 \mathrm{~dB}$ & $8.09 \mathrm{~dB}$ \\
\hline $\mathrm{GR}_{\text {no }} \mathrm{CL}_{\text {ind }}$ & $0.00 \mathrm{~dB}$ & $5.35 \mathrm{~dB}$ & $0.00 \mathrm{~dB}$ & $7.98 \mathrm{~dB}$ \\
\hline $\mathrm{GR}_{\text {agc }} \mathrm{CL}_{\max } \mathrm{QT}$ & $0.27 \mathrm{~dB}$ & $6.22 \mathrm{~dB}$ & $-2.25 \mathrm{~dB}$ & $6.99 \mathrm{~dB}$ \\
\hline $\mathrm{GR}_{\text {no }} \mathrm{CL}_{\max } \mathrm{QT}$ & $0.00 \mathrm{~dB}$ & $5.58 \mathrm{~dB}$ & $0.00 \mathrm{~dB}$ & $8.10 \mathrm{~dB}$ \\
\hline $\mathrm{GR}_{\text {no }} \mathrm{CL}_{\text {ind }} \mathrm{QT}$ & $0.00 \mathrm{~dB}$ & $5.46 \mathrm{~dB}$ & $0.00 \mathrm{~dB}$ & $8.03 \mathrm{~dB}$ \\
\hline WINNER II & $0.00 \mathrm{~dB}$ & $5.00 \mathrm{~dB}$ & $0.00 \mathrm{~dB}$ & $8.00 \mathrm{~dB}$ \\
\hline
\end{tabular}

TABLE III

VALUES FOR THE MEAN AND STANDARD DEVIATION OF THE SHADOW FADING OF ALL BSS FOR DIFFERENT ESTIMATION APPROACHES.

that experience approximately the same propagation conditions in terms of receive power and corresponding AGC adjustment. This leads to a higher estimation quality of the K-factor, as in the case of the DS. Important to note is that there are estimated values for the $\mathrm{K}$-factor despite the NLOS condition. This is due to the existence of several strong paths or strong clusters in the NLOS case.

\section{Cross Polarization Ratio (XPR)}

Table V summarizes the results for the vertical and horizontal XPR of base station 1. Both behave equivalent only with the difference that the values of the $\mathrm{XPR}_{\mathrm{h}}$ are in general about $2 \mathrm{~dB}-3 \mathrm{~dB}$ smaller in the NLOS case compared to the values of the $\mathrm{XPR}_{\mathrm{v}}$. The dependence on the QT is negligible and the deviation between AGC grouping and no AGC grouping is also very small.

\section{E. Cross correlation}

Table VI shows the cross correlation coefficients between the DS, SF and K-factor for NLOS and LOS with regard to the different estimation approaches. This coefficient states the degree of linear dependence between two variables. To turn the attention also to an other base station, BS2 is chosen for this evaluation. The DS and the SF are negatively correlated, which is in line with the results from [10], i.e., if the SF decreases together with the decreasing LOS component, the PDP is distributed more uniformly and therefore the DS increases. Additionally the values estimated considering the AGC grouping are less correlated. A possible explanation can be that due to the fact that the AGC grouping has almost no influence on the SF but on the DS hence the correlation decreases when the AGC dependent method is used. This entails that if the measurement is done with AGC switching and the following analysis is performed without considering the directional dependency the correlation might be overrated. The cross correlation coefficient for the DS and the K-factor

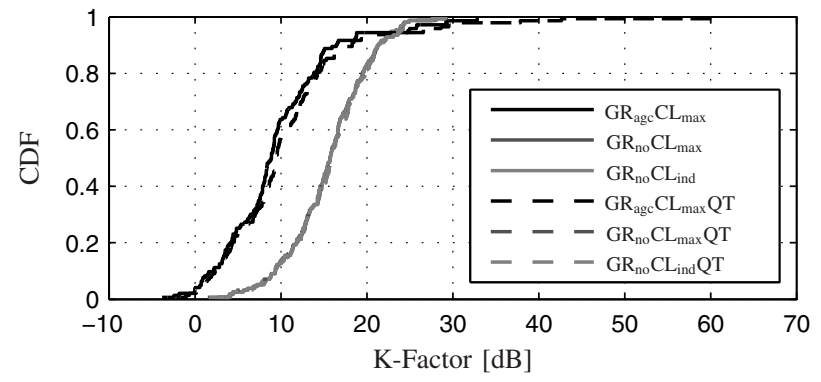

Fig. 3. $\mathrm{CDF}$ of the K-factor (LOS) of BS1 for different estimation approaches. 


\begin{tabular}{|l|c|c|c|c|}
\hline \multirow{2}{*}{} & \multicolumn{2}{|c|}{ NLOS } & \multicolumn{2}{c|}{ LOS } \\
\cline { 2 - 5 } & mean & std & mean & std \\
\hline \hline GR $_{\text {agc }} C_{\max }$ & $4.79 \mathrm{~dB}$ & $6.06 \mathrm{~dB}$ & $9.51 \mathrm{~dB}$ & $6.57 \mathrm{~dB}$ \\
\hline $\mathrm{GR}_{\text {no }} \mathrm{CL}_{\max }$ & $18.53 \mathrm{~dB}$ & $4.00 \mathrm{~dB}$ & $15.57 \mathrm{~dB}$ & $4.80 \mathrm{~dB}$ \\
\hline $\mathrm{GR}_{\text {no }} \mathrm{CL}_{\text {ind }}$ & $19.04 \mathrm{~dB}$ & $4.02 \mathrm{~dB}$ & $15.58 \mathrm{~dB}$ & $4.79 \mathrm{~dB}$ \\
\hline $\mathrm{GR}_{\text {agc }} \mathrm{CL}_{\max } \mathrm{QT}$ & $5.41 \mathrm{~dB}$ & $6.35 \mathrm{~dB}$ & $10.64 \mathrm{~dB}$ & $8.22 \mathrm{~dB}$ \\
\hline $\mathrm{GR}_{\text {no }} \mathrm{CL}_{\max } \mathrm{QT}$ & $18.67 \mathrm{~dB}$ & $4.02 \mathrm{~dB}$ & $15.67 \mathrm{~dB}$ & $4.88 \mathrm{~dB}$ \\
\hline $\mathrm{GR}_{\text {no }} \mathrm{CL}_{\text {ind }} \mathrm{QT}$ & $19.19 \mathrm{~dB}$ & $4.04 \mathrm{~dB}$ & $15.67 \mathrm{~dB}$ & $4.88 \mathrm{~dB}$ \\
\hline WINNER II & n.a. & $7.20 \mathrm{~dB}$ & n.a. & $3.40 \mathrm{~dB}$ \\
\hline
\end{tabular}

TABLE IV

VALUES FOR THE MEAN AND STANDARD DEVIATION OF THE K-FACTOR OF BS 1 FOR DIFFERENT ESTIMATION APPROACHES.

is also expected to be negative. The values of the $\mathrm{K}$-factor increases with the power of the LOS component. But therefore the DS decreases with the power of the NLOS components. Compared with the results in Table VI it affirms that the AGC dependent processing method is the more precise way for the estimation of the LSPs. Due to the measurement with AGC switching between the MIMO channels the analysis has also to be done in dependency of the AGC values. The correlation values for the $\mathrm{K}$-factor and the SF confirm this assumption. They have consequently to be positive, due to the negative correlation between DS and SF and between DS and K-factor. This occurs only for the AGC depending method, whereas the values for the non grouping approach are negative. The correlation coefficients for the K-factor in the NLOS case can be a little bit distorted because of the fact that the most powerful NLOS path or cluster is considered for the estimation of the parameter.

\section{CONCLUSION AND OUTLOOK}

Supporting the AGC switching within the measurement MIMO matrices by the channel sounding equipment is very beneficial and useful. The dynamic range of the single MIMO snapshot increases and hence a better identification of weaker multipath components is possible. This affords a more precise measurement. Therefore special care of the subsequent data analysis has to be taken. In particular in the case of directional antenna arrays, e.g. UCA with patch elements, the received multipath contributions and hence the AGC adjustments can change strongly dependent on the direction of the antenna patches among each other. In our measurement this directivity of the receive antenna dominates the influence of the fading. So a strong spatial filtering of the measured channel compared to omnidirectional elements can be expected. This implicates, that in further analysis and estimation, e.g. of LSPs of the WINNER channel model, the variations in the AGC values have to be inevitably taken into account. Within this paper the different approaches as AGC dependent grouping and no grouping are introduced and compared. It is shown that AGC grouping results in differences in the mean value and the standard deviation of the LSPs. It is discovered that this directional dependent analysis is more distinctive for the DS and the Kfactor, but less sensitive for the SF and the XPRs. Nevertheless when using AGC switching within the measurement MIMO matrices, an AGC value dependent analysis is indispensable. A second achievement is the influence of a QT which has not an effect on all parameters in equal measure. The values of

\begin{tabular}{|c|c|c|c|c|}
\hline & \multicolumn{4}{|c|}{$\mathrm{XPR}_{\mathrm{v}}$} \\
\hline & \multicolumn{2}{|c|}{ NLOS } & \multicolumn{2}{|c|}{ LOS } \\
\hline & mean & std & mean & std \\
\hline $\mathrm{GR}_{\mathrm{agc}} \mathrm{CL}_{\max }$ & $7.76 \mathrm{~dB}$ & $2.77 \mathrm{~dB}$ & $11.02 \mathrm{~dB}$ & $2.69 \mathrm{~dB}$ \\
\hline $\mathrm{GR}_{\mathrm{no}} \mathrm{CL}_{\max }$ & $7.26 \mathrm{~dB}$ & $2.63 \mathrm{~dB}$ & $10.87 \mathrm{~dB}$ & $2.65 \mathrm{~dB}$ \\
\hline $\mathrm{GR}_{\mathrm{no}} \mathrm{CL}_{\text {ind }}$ & $7.00 \mathrm{~dB}$ & $2.63 \mathrm{~dB}$ & $10.80 \mathrm{~dB}$ & $2.62 \mathrm{~dB}$ \\
\hline $\mathrm{GR}_{\mathrm{agc}} \mathrm{CL}_{\max } \mathrm{QT}$ & $7.78 \mathrm{~dB}$ & $2.75 \mathrm{~dB}$ & $11.01 \mathrm{~dB}$ & $2.70 \mathrm{~dB}$ \\
\hline $\mathrm{GR}_{\text {no }} \mathrm{CL}_{\max } \mathrm{QT}$ & $7.22 \mathrm{~dB}$ & $2.61 \mathrm{~dB}$ & $10.80 \mathrm{~dB}$ & $2.65 \mathrm{~dB}$ \\
\hline $\mathrm{GR}_{\mathrm{no}} \mathrm{CL}_{\text {ind }} \mathrm{QT}$ & $7.06 \mathrm{~dB}$ & $2.58 \mathrm{~dB}$ & $10.77 \mathrm{~dB}$ & $2.63 \mathrm{~dB}$ \\
\hline \multirow[t]{4}{*}{ WINNER II } & $7.00 \mathrm{~dB}$ & $3.00 \mathrm{~dB}$ & $8.00 \mathrm{~dB}$ & $4.00 \mathrm{~dB}$ \\
\hline & \multicolumn{4}{|c|}{$\mathrm{XPR}_{\mathrm{h}}$} \\
\hline & \multicolumn{2}{|c|}{ NLOS } & \multicolumn{2}{|c|}{ LOS } \\
\hline & mean & std & mean & std \\
\hline $\mathrm{GR}_{\mathrm{agc}} \mathrm{CL}_{\max }$ & $4.74 \mathrm{~dB}$ & $3.33 \mathrm{~dB}$ & $10.75 \mathrm{~dB}$ & $2.97 \mathrm{~dB}$ \\
\hline $\mathrm{GR}_{\text {no }} \mathrm{CL}_{\max }$ & $4.40 \mathrm{~dB}$ & $3.24 \mathrm{~dB}$ & $10.48 \mathrm{~dB}$ & $2.82 \mathrm{~dB}$ \\
\hline $\mathrm{GR}_{\mathrm{no}} \mathrm{CL}_{\text {ind }}$ & $4.21 \mathrm{~dB}$ & $3.17 \mathrm{~dB}$ & $10.40 \mathrm{~dB}$ & $2.80 \mathrm{~dB}$ \\
\hline $\mathrm{GR}_{\mathrm{agc}} \mathrm{CL}_{\max } \mathrm{QT}$ & $4.75 \mathrm{~dB}$ & $3.34 \mathrm{~dB}$ & $10.76 \mathrm{~dB}$ & $3.00 \mathrm{~dB}$ \\
\hline $\mathrm{GR}_{\mathrm{no}} \mathrm{CL}_{\max } \mathrm{QT}$ & $4.37 \mathrm{~dB}$ & $3.23 \mathrm{~dB}$ & $10.43 \mathrm{~dB}$ & $2.83 \mathrm{~dB}$ \\
\hline $\mathrm{GR}_{\mathrm{no}} \mathrm{CL}_{\text {ind }} \mathrm{QT}$ & $4.27 \mathrm{~dB}$ & $3.16 \mathrm{~dB}$ & $10.40 \mathrm{~dB}$ & $2.82 \mathrm{~dB}$ \\
\hline WINNER II & $7.00 \mathrm{~dB}$ & $3.00 \mathrm{~dB}$ & $8.00 \mathrm{~dB}$ & $4.00 \mathrm{~dB}$ \\
\hline
\end{tabular}

TABLE V

VALUES FOR THE MEAN AND STANDARD DEVIATION OF THE VERTICAL AND HORIZONTAL CROSS POLARIZATION RATIO OF BS1 FOR DIFFERENT ESTIMATION APPROACHES.

\begin{tabular}{|l|c|c|c|c|c|c|}
\hline & \multicolumn{2}{|c|}{ DS \& SF } & \multicolumn{2}{c|}{ DS \& K-factor } & \multicolumn{2}{c|}{ SF \& K-factor } \\
\cline { 2 - 7 } & NLOS & LOS & NLOS & LOS & NLOS & LOS \\
\hline \hline $\mathrm{GR}_{\mathrm{agc}} \mathrm{CL}_{\max }$ & -0.44 & -0.39 & -0.00 & -0.07 & 0.06 & 0.12 \\
\hline $\mathrm{GR}_{\mathrm{no}} \mathrm{CL}_{\max }$ & -0.52 & -0.66 & -0.09 & 0.30 & 0.07 & -0.13 \\
\hline $\mathrm{GR}_{\mathrm{no}} \mathrm{CL}_{\text {ind }}$ & -0.57 & -0.74 & 0.25 & 0.38 & -0.07 & -0.21 \\
\hline $\mathrm{GR}_{\mathrm{agc}} \mathrm{CL}_{\max } \mathrm{QT}$ & -0.32 & -0.30 & -0.01 & -0.11 & -0.00 & 0.18 \\
\hline $\mathrm{GR}_{\mathrm{no}} \mathrm{CL}_{\max } \mathrm{QT}$ & -0.31 & -0.65 & -0.00 & 0.00 & 0.08 & -0.08 \\
\hline $\mathrm{GR}_{\text {no }} \mathrm{CL}_{\text {ind }} \mathrm{QT}$ & -0.35 & -0.73 & 0.05 & 0.09 & 0.05 & -0.16 \\
\hline WINNER II & -0.4 & -0.4 & n.a. & -0.4 & n.a. & 0.3 \\
\hline
\end{tabular}

TABLE VI

CROSS CORRELATION COEFFICIENT OF DELAY SPREAD,SHADOW FADING AND K-FACTOR OF BS2 FOR DIFFERENT ESTIMATION APPROACHES.

the DS for example decrease whereas the values of the TL, SF or XPR are nearly the same. That suggests that the QT has only an influence on the delay domain but not on the power domain parameters. For further research the question is open, whether the mean or the maximum out of estimated LSPs within the AGC groups is the better selection for the parameter estimation and which influence has this decision on the distribution of the LSPs. Up to now the mean is considered for the DS, SF and XPRs but for the K-factor the maximum is used. A future workplan is to turn the attention on how the parameters behave with regard to the different polarizations.

[1] http://www.ist-winner.org

\section{REFERENCES}

[2] http://www.medav.de

[3] M. Narandzic, M. Landmann, C. Schneider, R.S. Thomä, "Influence of Extraction Procedures on Estimated Wideband MIMO Channel Parameters", IST Mobile and Wireless Comm. Summit, Hungary, 2007.

[4] http://www-emt.tu-ilmenau.de/ReferenceScenario/

[5] C. Schneider, G. Sommerkorn, M. Narandzic, M. Käske, A. Hong, V. Algeier, W. Kotterman, R. Thomä, C. Jandura, 'Multi-User MIMO Channel Reference Data for Channel Modelling and System Evaluation from Measurements", Int. IEEE WSA, Berlin, Germany, 2009.

[6] http://www.3gpp.org

[7] A. Boettcher, C. Schneider, M. Narandzic, P. Vary, R.S. Thomae, "Power and Delay Domain Parameters of Channel Measurements at $2.53 \mathrm{GHz}$ in an Urban Macro Cell Szenario", EuCAP, Barcelona, Spain, 2010.

[8] L.J. Greenstein, D.G. Michelson, V. Erceg, 'Moment method estimation of the Ricean K-factor", IEEE Comm. Letters, Vol.3, Issue 6, 1999.

[9] T. S. Rappaport, Wireless Comm.: Principles and Practice, 2nd Edition, Prentice Hall, 2002.

[10] L.J. Greenstein, V. Erceg, Y.S. Yeh, M.V. Clark, "A New PathGain/Delay-Spread Propagation Model for Digital Cellular Channels", IEEE Transactions on Vehicular Technology, Vol.46, No.2, 1997. 